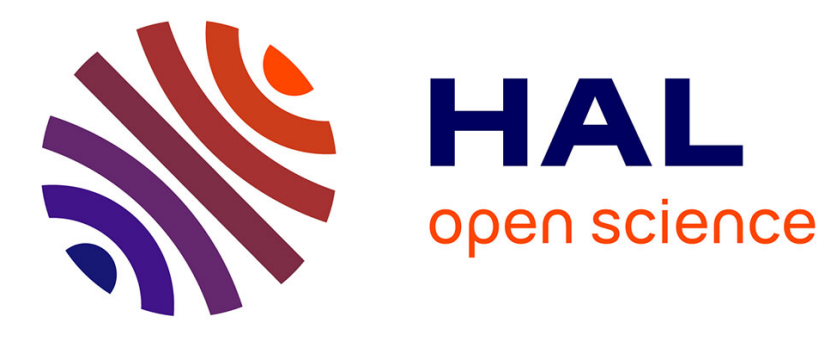

\title{
Sur un exemple de propagation dans un milieu désordonné
}

\author{
P.-G. de Gennes, P. Lafore, J.P. Millot
}

\section{To cite this version:}

P.-G. de Gennes, P. Lafore, J.P. Millot. Sur un exemple de propagation dans un milieu désordonné. J. Phys. Radium, 1959, 20 (6), pp.624-632. 10.1051/jphysrad:01959002006062400 . jpa-00236110

\section{HAL Id: jpa-00236110 https://hal.science/jpa-00236110}

Submitted on 1 Jan 1959

HAL is a multi-disciplinary open access archive for the deposit and dissemination of scientific research documents, whether they are published or not. The documents may come from teaching and research institutions in France or abroad, or from public or private research centers.
L'archive ouverte pluridisciplinaire HAL, est destinée au dépôt et à la diffusion de documents scientifiques de niveau recherche, publiés ou non, émanant des établissements d'enseignement et de recherche français ou étrangers, des laboratoires publics ou privés. 


\title{
SUR UN EXEMPLE DE PROPAGATION DANS UN MILIEU DÉSORDONNÉ
}

\author{
Par P. G. DE GENNES, P. LAFORE et J. P. MILLOT, \\ Centres d'Études Nucléaires de Saclay et de Fontenay-aux-Roses.
}

\begin{abstract}
Résumé. - On envisage une solution solide où des atomes " actifs " A sont substitués, au hasard aux nœuds d'une matrice inactive $B$. On étudie un phénomène de propagation qui fait seulement intervenir les atomes $\mathrm{A}$. Ce cas se rencontre notamment pour les bandes d'impuretés des semiconducteurs, et pour les ondes de spin d'un alliage à un constituant ferromagnétique.

Nous montrons ici comment certaines propriétés géométriques de la répartition des diffuseurs, qui ont été étudiées précédemment, jouent un rôle essentiel sur : a) le spectre de valeurs propres de l'équation d'ondes ; $b$ ) les phénomènes de transport.
\end{abstract}

Abstract. - This paper deals with propagation phenomena in binary solid solutions $A B$, the waves being carried by the A atoms only. Such a situation is met in impurity bands en semiconductors, and also for spin waves in alloys with one ferromagnetic component. We here investigate the influence of accidental clusters formed by $\mathrm{A}$ atoms on the eigenvalue spectrum and on the transport properties.

I. Introduction. - Soit une solution solide $\mathrm{AB}$, où des atomes " actifs" A sont substitués au hasard, aux nœuds $(i)$ du réseau périodique de la matrice $B$. Deux exemples nous serviront à préciser ce que nous entendons par atomes " actifs".

1 er exemple: Les atomes A sont porteurs de spins $S$, et couplés ferromagnétiquement entre eux par une énergie d'échange. La matrice $B$ est, elle, magnétiquement inactive. La question de savoir dans quelles conditions un tel alliage admet un point de Curie bien défini a été soulevée par J. Friedel. On peut y apporter une réponse au moins partielle, en étudiant, aux basses températures, la stabilité de l'ordre ferromagnétique à l'égard des premières excitations de spin. Il faut donc déterminer les niveaux d'onde de spin de l'alliage désordonné. Ce sont les valeurs propres $E$ de l'équation de propagation :

$$
E a_{i}=2 \sum_{j}^{\prime} a_{j} J_{i j}
$$

où $a$ représente l'amplitude de la déviation du $i^{\mathrm{e}}$ spin, et où la somme $\Sigma_{j}^{\prime}$ est limitée aux sites qui sont également occupés par des atomes $\mathrm{A}$.

$2^{\mathrm{e}}$ exemple : Les atomes A forment une " bande d'impuretés " dans un semi-conducteur. A chaque atome $\mathrm{A}$, situé au site (i), est associé une orbitale $\varphi_{i}(R)$, et on cherche, dans l'approximation des liaisons fortes, des fonctions d'onde à un électron de la forme :

$$
\psi(\boldsymbol{R})=\sum_{i} a_{i} \varphi_{i}(\boldsymbol{R}) .
$$

Si les $\varphi_{i}$ relatives à des sites différents sont orthogonales, on a encore pour les $a_{i}$ une équation du type (1).
Dans la suite, nous nous limiterons au cas où seuls les premiers voisins sont couplés entre eux : cette simplification correspond mal aux situations physiques usuelles, mais elle sera utile pour mettre en relief les résultats essentiels (ceux-ci ne sont pas modifiés qualitativement tant que la portée des couplages reste finie). Par un choix convenable de l'échelle des énergies, (1) peut alors être écrite :

$$
E a_{i}=\Sigma_{j i}^{\prime \prime} a_{j}
$$

où la somme $\Sigma_{j}^{\prime \prime}$ est limitée aux premiers voisins, occupés par des atomes A, de l'atome A. C'est à cette forme réduite que nous nous intéresserons dans la suite.

Lorsque tous les nœuds du réseau sont occupés par des atomes A, les solutions de (3) sont des ondes planes $a_{i}=\mathrm{e}^{i k . R_{i}}$ (où $R_{i}$ est la coordonnée du Ier Site) et les niveaux correspondants sont :

$$
E(\boldsymbol{k})=\Sigma_{j}^{(\mathbf{1})} \mathrm{e}^{i \mathbf{k} \cdot\left(\mathbf{R}_{j}-\mathbf{R}_{i}\right) .}
$$

Dans (4) la somme $\Sigma^{(1)}$ est étendue à tous les premiers voisins d'un site quelconque $\left.(i){ }^{1}\right)$. Le fait essentiel est que ces niveaux forment un spectre continu. Introduisons maintenant des atomes B. Les premiers termes du développement de la densité de niveaux en série de puissances de la concèntration en atomes B, sont convergents (section II) ; ils représentent une simple déformation du spectre continu.

$\mathrm{Au}$ contraire, aux faibles concentrations en atomes A, ceux-ci sont pour la plupart isolés. Il leur est associé un seul niveaux $E=0$ fortement dégénéré. Quelques atomes $\mathrm{A}$ forment des paires et donnent deux niveaux $E= \pm 1$. Quelques-uns

(1) Nous nous limitons à des réseaux de Bravais. 
encore forment des triplets et donnent trois niveaux, etc... La série ainsi engendrée converge rapidement dans ce domaine (voir section III) et le spectral global est discret.

Que se passe-t-il dans le domaine intermédiaire ? Chaque atome $\mathrm{A}$ appartient à un " amas " de $n$ atomes A. L'équation de propagation ne couple que les atomes d'un même amas. Le rang $n$ de l'amas peut être fini ou infini. Les amas infinis jouent un rôle à part : ils contribuent seuls au spectre continu et aux phénomènes de transport sur des distances macroscopiques. Il est done essentiel de déterminer le pourcentage d'atomes A engagés dans des amas infinis : deux méthodes d'approximation sont appliquées à ce problème dans la section III. Enfin, dans la section IV, on indique comment appliquer ees résultats à l'étude du spectre ou de la conductivité aux hautes températures, grâce à une méthode de moments.

II. Fortes concentrations en atomes actifs. Soit $N$ le nombre de sites du cristal, $N x$ le nombre d'atomes A répartis au hasard), $N y=N(1-x)$ le nombre d'atomes $B$. Nous nous intéressons ici aux faibles valeurs de $y$ : nous utiliserons done une technique de perturbation, l'hamiltonien non perturbé étant celui du cristal A pur :

$$
\begin{aligned}
\left\langle i\left|\varkappa_{0}\right| j\right\rangle & =1 \text { pour } i \text { et } j . \text { voisins } \\
& =0 \text { dans tous les autres cas. }
\end{aligned}
$$

Ses niveaux sont donnés par (4) et ses vecteurs propres $\mid k>$ sont définis par :

$$
\langle i \mid \boldsymbol{k}\rangle=N^{-1 / 2} \mathrm{e}^{\mathbf{i} \mathbf{k} \cdot \mathbf{R}_{i}} \text {. }
$$

La perturbation introduite par les atomes $B$ peut être décrite comme un potentiel infiniment répulsif agissant sur les sites $B$

$$
\left\langle i\left|x-x_{0}\right| j\right\rangle=V \delta_{i j}
$$

pour $(i)$ occupé par un atome $B$, et nul dans le cas contraire. $V$ est un nombre positif que nous ferons tendre vers l'infini à la fin du calcul, ce qui assurera la-nullité de l'amplitude au site correspondant $\left({ }^{2}\right)$.

La densité de niveaux dans la solution solide est donnée par :

$$
N \rho(E)=\overline{\text { Trace }\{\delta(E-x)\}}
$$

(2) Le lecteur attentif sera peut être surpris par notre définition (7) de la perturbation. On est tenté de prendre en effet $\left\langle i\left|x-x_{0}\right| j\right\rangle=-1$ pour $(i)$ et $(i)$ voisins, (i) ou (j) étant un atome B, et nul dans tous les autres cas. Avec ce potentiel, on trouve, au lieu de (13),

$$
\begin{aligned}
& <\boldsymbol{k}\left|T_{i}(E)\right| \boldsymbol{l}> \\
& \quad=N^{-1} \mathrm{e}^{i(1-\mathbf{k}) \mathbf{R}_{i}}\left[T(E)+\frac{(E-E(\boldsymbol{k}))(E-E(\boldsymbol{l}))}{E}\right] .
\end{aligned}
$$

Cette modification est due à l'existence d'un niveau parasite $(E=0)$ sur l'atome $B$, qui est automatiquement éliminé par (7). la moyenne étant prise sur toutes les configurations possibles des atomes A et B. Introduisons l'opérateur $\tilde{T}(E)$ défini par :

$$
\tilde{T}(E)=\left[\varkappa-\varkappa_{0}\right]\left[1+\left(E-\varkappa_{0}+i \varepsilon\right)^{-1} \tilde{T}(E)\right]
$$

on a :

$$
\begin{aligned}
\rho(E)= & \frac{i}{2 \pi N}\left[\operatorname{Trace}\left\{\frac{1}{E+i \varepsilon-\chi}\right\}-C C\right] . \\
= & \rho_{\theta}(E)+\frac{i}{2 \pi N} \sum_{\mathbf{k}}(E+i \varepsilon-E(\boldsymbol{k}))^{-\boldsymbol{\varepsilon}} \\
& {[<\boldsymbol{k}|\tilde{T}(E)| \boldsymbol{k}>-C C] . }
\end{aligned}
$$

Soit $\tilde{T}_{i}(E)$ l'opérateur auquel se réduit $\tilde{T}(E)$ lorsqu'il existe un seul atome $B$ dans le réseau, au site $(i) . \quad \tilde{T}(E)$ peut être exprimé en fonction des $\tilde{T}_{i}(E)$ par l'équation de Watson [1].

$$
\tilde{T}=\sum_{L} \tilde{T}_{j}+\sum_{i \neq j} \tilde{T}_{L} D^{-1} \tilde{T}_{j}+\sum_{i \not p j \neq k} \tilde{T}_{i} D^{-1} \bar{T}_{j} D^{-1} \bar{T}_{k}
$$

où a) les sommations sont étendues aux sites occupés par les atomes $\mathrm{B}, b$ ) deux indices successifs sont toujours différents, $c$ ) on a posé

$$
D=\left(E+i \varepsilon-x_{0}\right) \text {. }
$$

D'autre part, on calcule facilement $\tilde{T}_{i}(E)$ grâce à la forme spéciale du potentiel perturbateur (7)

$$
\begin{aligned}
& \left.<\boldsymbol{k}\left|\tilde{T}_{i}(E)\right| \boldsymbol{l}\right\rangle \\
& \quad=N^{-1} \mathrm{e}^{i(1-\mathbf{k}) \cdot \mathbf{R}_{i}} \frac{V}{1-V N^{-1} \sum_{\mathbf{m}}\left\langle\boldsymbol{m}\left|D^{-1}\right| \boldsymbol{m}\right\rangle} .
\end{aligned}
$$

Nous prenons comme convenus la limite $V \rightarrow \infty$ et obtenons

$$
\left\langle\boldsymbol{k}\left|\tilde{T}_{i}(E)\right| \boldsymbol{l}>=N^{-1} \mathrm{e}^{i(\mathbf{l}-\mathbf{k}) \cdot \mathbf{n}_{i}} T(E)\right.
$$

avec

$$
[T(E)]^{-1}=-V_{0}(2 \pi)-3 \int_{\text {zone }} \mathrm{d}_{3} m \frac{1}{E+i \varepsilon-E(m)}
$$

$V_{0}$ désigne le volume atomique et l'intégrale est étendue à la première zone de Brillouin du réseau réciproque.

Nous pouvons maintenant effectuer la moyenne de (11) sur toutes les configurations possibles des atomes $\mathrm{A}$ et $\mathrm{B}$. Au premier ordre en $y$, seule la première somme $\sum_{i} \tilde{T}_{i}$ contribue. En reportant dans la densité de niveaux (10) on trouve :

$$
\begin{aligned}
\rho(E)-\rho_{0}(E) & =\frac{i y}{2 \pi}\left\{T(E) V_{0}(2 \pi)-3\right. \\
& \left.\int_{\text {zone }} d_{3} m \frac{1}{[E+i \varepsilon-E(m)]^{2}}-C C\right\}+0 . \mathrm{y} \\
= & =\frac{y}{\pi} \operatorname{Im} \cdot\left\{\frac{1}{T(E)} \frac{d T(E)}{d E}\right\}+0 . \mathrm{y}
\end{aligned}
$$


Ce résultat peut être transformé en examinant la diffusion par un atome $\mathrm{B}$ unique (pris à l'origine). La solution en ondes sortantes s'écrit :

$$
\begin{gathered}
a_{i}=\mathrm{e}^{i \mathbf{k} \cdot \mathbf{R}_{i}-\Gamma_{E}^{+}\left(\boldsymbol{R}_{i}\right)} \\
\Gamma_{E}^{+}\left(\boldsymbol{R}_{i}\right)=-T(E) V_{0}(2 \pi)^{-3} \int_{\text {zone }} d_{3} m \frac{\mathrm{e}^{i \mathbf{m} \cdot \mathbf{R}_{i}}}{E+i \varepsilon-E(\boldsymbol{m}} \cdot
\end{gathered}
$$

L'onde sortante a toutes les symétries du groupe ponctuel : c'est l'analogue réticulaire d'une onde $s$, Le déphasage $\delta$ correspondant est donné par [2]

$$
\pi T(E) \rho_{0}(E)=\mathrm{e}^{i \delta} \sin \delta .
$$

En reportant cette valeur dans (15) on obtient

$$
\rho(E)-\rho_{0}(E)=\frac{y}{\pi} \frac{\mathrm{d} \delta}{\mathrm{d} E} .
$$

C'est un cas particulier d'une formule due à Friedel pour les milieux continus [3] et généralisé par de Witt [4]. Il est également possible de montrer que, aux grandes longueurs d'ondes (18) est équivalent à la relation classique entre l'indice de réfraction $n$ et la longueur de diffusion par un atome $\mathrm{B}$, soit $a$,

$$
n^{2}-1=\frac{y}{V_{0}} \frac{a \lambda^{2}}{\pi} .
$$

On en déduit également que le niveau le plus haut, dans cette approximation est

$$
\begin{gathered}
E_{\max }=E(0)-y K(0) \\
{[K(E)]^{-1}=V_{0}(2 \pi)^{-3} \int_{\text {zone }} d_{3} m \frac{\mathscr{P}}{E^{\prime}-E^{\prime}(m)}}
\end{gathered}
$$

ou le symbole $\mathscr{P}$ représente une partie principale au sens de Cauchy. La relation (20) est liée à certaines propriétés combinatoires remarquables des réseaux périodiques (voir appendice 1).

Passons maintenant à l'étude des termes en $y^{2}$ dans la densité de niveaux. Pour les sommer exactement, il faut regrouper dans (11) tous les termes faisant intervenir deux atomes B situés en $(i)$ et $(j)$ : soit $\tilde{T}_{i j}(E)$ la contribution correspondante. On peut sommer l'élément diagonal de cet opérateur en utilisant (11) et (13) :

$<\boldsymbol{k}\left|T_{y}(E)\right| \boldsymbol{k}>$

$=N^{-1} T(E)\left\{\frac{1-\Gamma_{E}^{+}\left(\boldsymbol{R}_{j}-\boldsymbol{R}_{i}\right) \mathrm{e}^{i \mathbf{k}\left(\mathbf{R}_{L}-\mathbf{R}_{j}\right)}}{1-\Gamma_{E}^{+}\left(\boldsymbol{R}_{j}-\boldsymbol{R}_{i}\right) \Gamma_{E}^{+}\left(\boldsymbol{R}_{i}-\boldsymbol{R}_{j}\right)}+\right.$ sym. $\}$.

Le terme symétrique étant déduit du précédent par l'échange de $i$ et $j$. (Sur la couche d'énergie $E=E(\boldsymbol{k})$, on aurait pu obtenir (22) par un calcul direct de l'onde diffusée.) On obtient ainsi :

$$
\begin{aligned}
<\boldsymbol{k}|\tilde{T}(E)| \boldsymbol{k}>=y T(E) \\
\quad+\frac{y^{2}}{2} \sum_{i \neq 0}\left\{\frac{1-\Gamma_{E}^{+}\left(\boldsymbol{R}_{i}\right) \mathrm{e}^{-i \mathbf{k} \cdot \mathbf{R}_{i}}}{1-\Gamma_{E}^{+}\left(\boldsymbol{R}_{i}\right) \Gamma_{E}^{+}\left(-\boldsymbol{R}_{i}\right)}+\text { sym. }-2\right\} .
\end{aligned}
$$

Le fait important est que la somme qui figure dans (23) converge : pour $|\boldsymbol{R}| \rightarrow \infty$, à trois dimensions, $\Gamma_{E}^{+}(\boldsymbol{R})$ décroît comme $1 / \boldsymbol{R}$. Les termes qui devront être examinés séparément sont :

$$
\begin{gathered}
-\sum_{i \neq 0} \Gamma_{E}^{+}\left(\boldsymbol{R}_{i}\right) \mathrm{e}^{-i \mathbf{k} \cdot \mathbf{R}_{i}=1+\frac{T(E)}{E-E(\boldsymbol{k})+i \varepsilon}} \\
\sum_{i \neq 0} \Gamma_{E}^{+}\left(\boldsymbol{R}_{i}\right) \Gamma_{E}^{+}\left(-\boldsymbol{R}_{i}\right)=-1-\frac{\mathrm{d} T}{\mathrm{~d} E} \text { etc... }
\end{gathered}
$$

On voit qu'ils sont finis, et, en reportant dans (10), que le terme en $y^{2}$ de la densité de niveaux est convergent. Jusqu'à l'ordre $y^{2}$, on obtient donc un spectre déformé, mais qui reste essentiellement continu. Cet état de choses ne sera en fait modifié que par l'apparition d'états liés, dus aux atomes A qui sont complètement entourés par des atomes B. La première contribution de ces amas finis correspond à un atome $\mathbf{A}$ isolé par $\boldsymbol{Z}$ voisins B ; elle est donc d'ordre $y^{Z}$. Pour éliminer la contribution de ces amas finis, nous allons en premier lieu, dans la section suivante, estimer le pourcentage d'atomes A engagés dans de tels amas.

Il n'est pas inutile de souligner enfin les différences qui existent entre le cas tridimensionnel envisagé ci-dessus et celui d'une chaîne linéaire : pour une chaîne linéaire $T(E)$ est imaginaire pur (il n'y a pas de propagation cohérente) et le spectre est discret sur tout l'intervalle de concentration $0 \leqslant x<1$.

III. Étude géométrique des amas. - Faisons choix d'un site origine 0 (quelconque) et soit $P n(x)$ la probabilité pour que 0 soit occupépar un atomeA qui appartiennent à un amas de rang $n$. Nous poserons par convention

$$
P_{0}(x)=y=1-x .
$$

Les probabilités $P n(x)$ pour $n$ fini se calculent de la façon suivante : pour chaque forme possible d'amas contenant 0 , on détermine : $a$ ) le rang $n$ de l'amas, $b$ ) le nombre $m$ d'atomes B qui sont voisins d'au moins un atome $\mathrm{A}$ de l'amas. (Ces sites $m$ constituent l'adhérence de l'amas.) Soit $A_{n m}$ le nombre total d'amas qu'il est possible de réaliser, contenant 0 , de rang $n$ et d'adhérence formée de $m$ atomes $\mathrm{B}$. On a

$$
P_{n}(x)=\sum_{\dot{m}} A_{n m} x^{n} y^{m} .
$$

Les facteurs $y$ spécifient que tous les points de l'adhérence sont bien des atomes B. Nous donnons

\section{TABLE 1}

$$
\text { 1. Réseau cubique simple. }
$$

$$
\begin{aligned}
P_{1} & =x y^{6} \\
P_{2} & =6 x^{2} y^{10} \\
P_{3} & =36 x^{3} y^{13}+9 x^{3} y^{14} \\
P_{4} & =x^{4}\left(32 y^{15}+204 y^{16}+96 y^{17}+12 y^{18}\right) \\
P_{5} & =x^{5}\left(60 y^{17}+495 y^{18}+1140 y^{19}+780 y^{20}\right. \\
& \left.+180 y^{21}+15 y^{22}\right)
\end{aligned}
$$




\section{Réseau Gubique centré.}

$P_{1}=x y^{8}$

$P_{2}=8 x^{2} y^{14}$

$P_{3}=x^{3}\left(12 y^{20}+36 y^{19}+36 y^{17}\right)$

3. Réseau Gubique a fages centrées.

$P_{1}=x y^{12}$

$P_{2}=12 x^{2} y^{18}$

$P_{3}=x^{3}\left(24 y^{23}+126 y^{24}\right)$

dans la table I les valeurs des $P n(x)$ (pour les premiers ordres) relatives à quelques types de réseaux.

La probabilité pour que 0 appartienne à un amas infini d'atomes A est obtenue par différence

$$
P_{\infty}(x) \equiv S(x)=1-\sum_{0}^{\infty} P_{n}(x) .
$$

En limitant dans (28) la somme à ses premiers termes, on obtient une valeur approchée par excès de $S(x)$. L'aspect des courbes ainsi obtenues ( $f i g .1)$

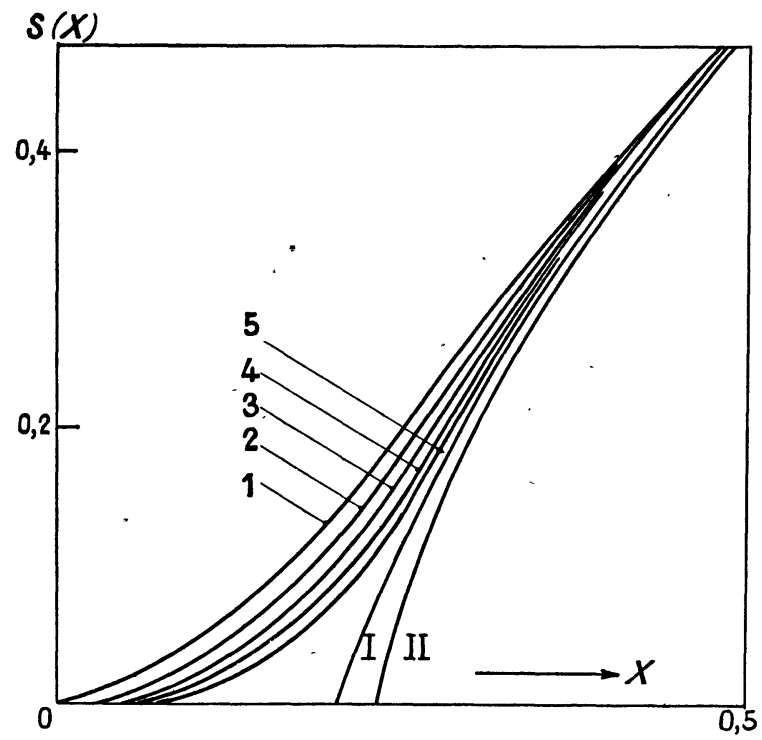

FIG. 1.

suggère l'existence de deux domaines de concentration dans lesquels le comportement de $S(x)$ diffère. Aux fortes concentrations (pratiquement pour $x>0.5) S(x)$ est très voisin de $x$ d'après la figure 1. Presque tous les atomes A sont engagés dans un même amas infini et la série (28) converge rapidement. (Toutefois, nous ne sommes pas parvenus à démontrer la convergence uniforme de (28) dans ce domaine).

Aux faibles concentrations $S(x)$ est identiquement nulle. En effet : a) $S(x)$ et ses dérivées convergent uniformément sur un segment fini $\left(\mathrm{O} x_{\mathbf{c}}\right)$. Pour un réseau cubique simple par exemple, l'adhérence $m$ est inférieure à $4 n+2$ (cette valeur étant obtenue par un amas : en forme de chaîne linéaire)

$$
P_{n}(x)>\left(\sum_{m} A_{n m}\right) x^{n} y^{\underline{a} n+2}
$$

la série $\sum_{n} P_{n}$ converge (puisque sa somme est bornée par I) et la valeur maxima de $x y^{\mathbf{4}}$ est $4^{4} / 5^{5}$. Donc $\left(\sum_{m} A^{m n}\right)$ est majoré par le terme de rang $n$ d'une série géométrique de raison $5^{5} / 4^{4}$, ce qui suffit à montrer la propriété.

b) Les dérivées $\left(\frac{\mathrm{d}^{n}}{\mathrm{~d} x^{n}} S\right)_{x=0}$ sont toutes nulles ; Pour la dérivée d'ordre $n$, seuls interviennent les amas de rang $q \leqslant n$ : subtituons donc au cristal infini un cristal fini de $f_{n}$ atomes, assez grand pour contenir tous les amas de rang $q \leqslant n$ et leurs adhérences. Soient $P_{q}^{\prime}$ les probabilités qui se substituent aux $P_{q}$ dans ces conditions

$$
\begin{gathered}
\sum_{0}^{f} P_{q}^{\prime}=1 \\
P_{q}^{\prime}=P_{q} \text { pour } q \geqslant n
\end{gathered}
$$

done

$$
\begin{aligned}
\left(\frac{\mathrm{d}^{n}}{\mathrm{~d} x^{n}} S\right)_{x=0} & =\left[\frac{\mathrm{d}^{n}}{\mathrm{~d} x^{n}} \sum_{0}^{n} P_{q}(x)\right]_{x=0}=\left[\frac{\mathrm{d}^{n}}{\mathrm{~d} x^{n}} \sum_{0}^{n} P_{q}^{\prime}(x)\right]_{x=0} \\
& =\left[\frac{\mathrm{d}^{n}}{\mathrm{~d} x^{n}} \sum_{0}^{f} P_{q}^{\prime}(x)\right]_{x=0}=0 .
\end{aligned}
$$

Donc sur un segment fini $\left(\mathrm{O} x_{o}\right), S(x)$ est égale à $S(0)$, donc est nulle : il n'y a pas d'amas infinis dans ce domaine de concentration.

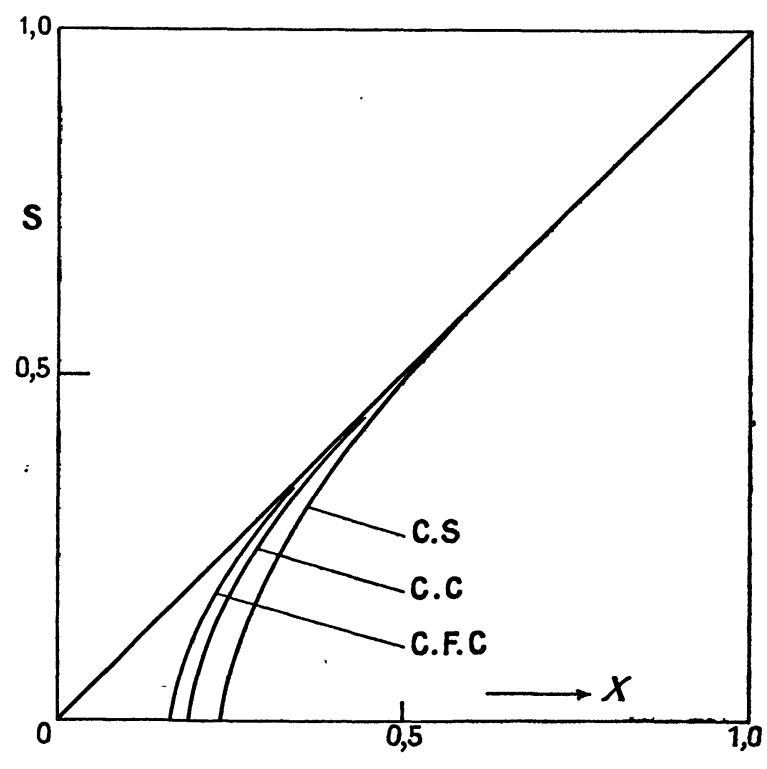

FIG. 2. 
Nous allons maintenant substituer à (28) un développement approché qui lui est identique aux premiers termes, que nous pouvons sommer, et qui fait apparaitre directement un " point critique " $x_{c}$. La méthode consiste à dénombrer les premiers amas construits à partir de 0 , puis à itérer la construction de ces amas pour en obtenir de plus grands. L'itération est approchée, car elle n'exclut pas certains recouvrements entre les parties successives de l'amas ou de l'adhérence. Nous en exposerons le principe sur le réseau cubique simple, et nous citerons simplement les résultats pour d'autres réseaux.

Posons

$$
\begin{aligned}
F(x)= & \sum_{0}^{\infty} P_{n}(x)=1-S(x) \\
= & y+x[G(x y)]^{6} \\
& (y=1-x) .
\end{aligned}
$$

Dans (31) nous avons séparé le cas où 0 est un atome $\mathrm{B}$, et la fonction $G(x y)$ doit engendrer des amas à partir d'un premier voisin $P_{1}$ de 0 , sachant que 0 et $P_{1}$ sont des atomes $\mathrm{A}$.

On écrit donc dans la première approximation

$$
G=y+x G^{5} .
$$

(L'exposant 5 au lieu de 6, tient compte du fait que 0 est déjà un atome A.) Dans la deuxième approximation, on remplace (32) par un système d'équations, destiné à construire au mieux les amas de trois atomes A. Ici, ces amas sont de deux types, selon que les trois points sont alignés ou forment un angle droit. Pour engendrer les amas à partir de $\boldsymbol{P}_{2}$ (fig. 3 ) sachant que 0 et $\boldsymbol{P}_{1}$ sont des

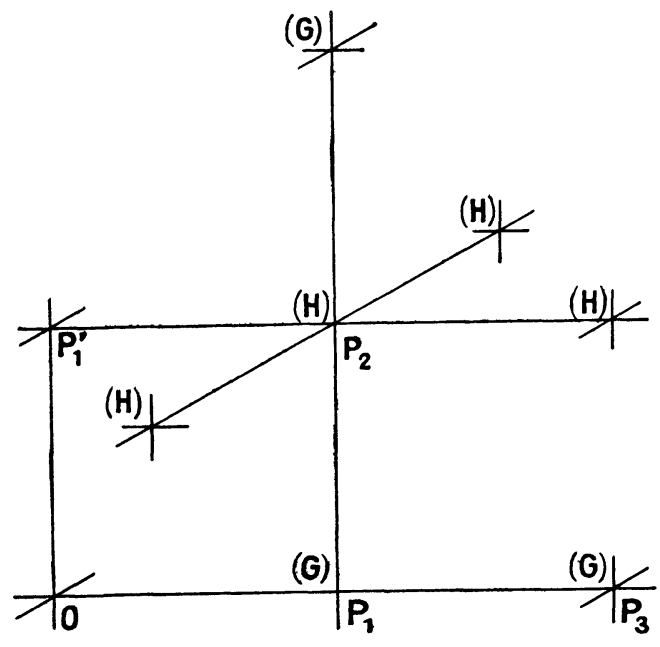

Fig. 3.

atomes $\mathbf{A}$, nous introduirons une nouvelle fonction $H(x y)$. Par contre, nous engendrons ceux qui sont issus de $P_{3}$, nous utilisons encore $G$. (En effet, la présence d'un atome $\mathbf{A}$ en $\mathrm{O}$ ne limite la construction des amas à partir de $P_{3}$ que pour des rangs élevés.)

On pose donc

$$
G=y+x G H^{4} .
$$

Il faut maintenant une équation pour $H$. Lorsque $0, P_{1}, P_{2}$ sont des atomes $A$, il reste seulement 4 sites voisins de $P_{2}$ à explorer ( $P_{1}^{\prime}$ a été exploré en même temps que $P_{1}$ grâce au facteur $G^{*}$ dans (31)). Pour ces 4 sites on néglige, comme pour $\boldsymbol{P}_{\mathbf{3}}$, le fait que 0 est occupé, ce qui donne

$$
H=y+x G H^{3}
$$

(31) et (32) ou bien (31), (33) et (34) déterminent $F$ en fonction de $x$ et $y=1-x$ dans la $1^{\text {re }}$ et dans la $2^{\mathbf{e}}$ approximation respectivement. (Les systèmes correspondants pour différents réseaux sont regroupés dans la table II.)

TABLE 2

$$
1^{\text {re approximation } 2 \text { approximation }}
$$

Cubique simple : $\quad G=y+x G^{5} \quad G=y+x H^{4} G$

$H=y+x H^{3} G$

Cubique face

$$
\text { centrée: } \quad G=y+x G^{7} \quad G=y+x G^{5} H^{2}
$$

Cubique centré : $\quad G=y+\approx G^{7} \quad G^{\gamma}=y+x G H_{1}^{3} H_{2}^{2}$

$H_{1}=y+x G H_{1}^{\prime} H_{2}$

$H_{2}=y+x G H_{1}^{3} H_{2}^{2}$

Si l'on voulait réaliser des approximations d'ordre supérieur, il ne faudrait pas seulement modifier le système qui donne $G$, mais aussi l'équation (31). Que peut-on déduire en pratique des système de la table II ? On vérifie d'abord, en développant la solution en série de $x$ et $y$ considérés comme variables indépendantes, que les premiers termes du développement (30) sont correctement reproduits, et que les termes suivants sont peu différents numériquement de la valeur exacte.

D'autre part, quelque soit le réseau (tridimensionnel) et l'ordre d'approximation (1 ou 2) lorsque l'on fait $y=1-x$ chaque système admet pour $F(x)$ deux solutions réelles sur l'intervalle (1), dont l'une est $F=1$, et dont l'autre est telle que $F(1)=0$.

Les deux déterminations se coupent pour une valeur $x_{c}$ de l'intervalle 01 . Partant de $x=0$ on doit d'abord suivre la première, puis, à partir de $x$, la seconde détermination. Les résultats sont représentés sur les figures 1 et 2 . On suit clairement, grâce à cette approximation, l'apparition des amas infinis. Les points critiques $x_{0}$ estimés de cette façon sont

$$
\begin{aligned}
x_{c}= & 0,224 \\
& 0,192 \\
& 0,162
\end{aligned}
$$


pour les réseaux cubiques simple, centré et face centrée respectivement.

Mentionnons ici encore les propriétés particulières de la chaîne linéaire : Pour tout $x \neq 1$ il n'y a que des amas finis d'atomes A. $S(x)$ est nul pour $x<1$ et discontinu pour $x=1$. Ceci est dû au fait qu'un nombre même faible, d'atomes B répartis sur la chaîne, suffit à la couper en segments finis, et se relie directement à l'absence de propagation cohérente signalée dans la section II.

IV. Principe de la séparation du spectre continu. - Nous avons vu comment, dans le domaine des fortes concentrations en atomes $\mathrm{A}$, une généralisation de l'approximation "optique " appliquée à la diffusion par les atomes $B$ permet de eéterminer le spectre perturbé. La conductivité dans la bande correspondante pourrait être calculée par les mêmes méthodes. Dans le domaine des faibles concentrations, le spectre se réduit à quelques raies discrètes calculables directement et la conductivité statique, elle, est nulle. Il est plus particulièrement intéressant d'interpoler ces résultats aux concentrations intermédiaires. Nous indiquons ici le principe d'une méthode de moments qui permet d'y parvenir, et les résultats numériques des toutes premières approximations correspondantes.

L'hamiltonien $x$ associé à (3) n'a d'élément de matrice qu'entre atomes A voisins (et ils sont alors égaux à 1). Il est.facile de calculer ses moments

$$
\begin{aligned}
\overline{\overline{x^{n}}} & =\frac{\overline{\operatorname{Tr} x^{n}}}{\operatorname{Tr} 1}=\frac{1}{x} \int E^{n} \rho(E) \mathrm{d} E \\
& =\sum_{p} N_{n p}(0) x^{p} .
\end{aligned}
$$

$N_{n p}\left(\boldsymbol{R}_{i}\right)$ est le nombre de chemins reliant les sites (0) et (i) en $n$ sauts de $1^{\text {er }}$ en $1^{\text {er }}$ voisin, et qui utilisent en tout $p$ points. distincts du réseau (autres que 0 ). On peut se proposer de calculer à la main les premiers moments, puis de construire $\rho(E)$ à partir de ces moments en lui imposant une forme analytique plausible. Cette opération n'est relativement permise que lorsque $\rho(E)$ est une fonction continue et régulière de $E$; pour l'appliquer ici, il faut d'abord soustraire la contribution des amás finis aux moments.

D'une façon générale, étant donnée une quantité $Q$ supposée additive pour des amas disjoints, nous désignons par $Q_{\infty}$ la partie de $Q$ qui provient des amas in finis.

En premier lieu nous connaissons

$$
\int p_{\infty}(E) \mathrm{d} E=S(x) .
$$

Pour calculer $\overline{\bar{x}}_{\infty}^{2}$ il faut déterminer la probabilité $S_{2}(x)$ pour que deux voisins soient simultanément occupés par des atomes $A$ et qu'ils appar. tiennent à un même amas infini. $S_{2}(x)$ s'obtient comme $S(x)$, par différence :

$$
\begin{aligned}
& S_{2}(x)-x^{2}-x^{2} y^{13}-x^{3}\left(8 y^{18}+2 y^{14}\right) \\
& -x^{4}\left(8 y^{15}+52 y^{16}+24 y^{17}+3 y^{18}\right)
\end{aligned}
$$

(pour un cubique simple). $S_{2}(x)$ comme $S(x)$, est une série de polynômes qui représente deux fonctions différentes selon que $x$ est inférieur ou supérieur à une certaine valeur critique, d'ailleurs égale à $x_{\mathrm{c}}$ comme le montrent les inégalités :

$$
S(x) \geqslant S_{2}(x) \geqslant x S(x) .
$$

On peut aussi faire une sommation approchée de $S_{2}(x)$. Dans l'exemple ci-dessus, on trouve, par la deuxième approximation

$$
S_{2}(x)=x^{2}\left(1-G^{2} H^{8}\right) .
$$

On en tire ensuite le deuxième moment

$$
\begin{aligned}
\overline{\bar{\varkappa}}_{\infty}^{2} & =\frac{\int^{E^{2} \rho_{\infty}(E) \mathrm{d} E}}{\int \rho_{\infty} \mathrm{d} E} \\
& =z \frac{S_{2}(x)}{S(x)}
\end{aligned}
$$

où $Z=E(0)$ est le nombre de voisins d'un site donné.

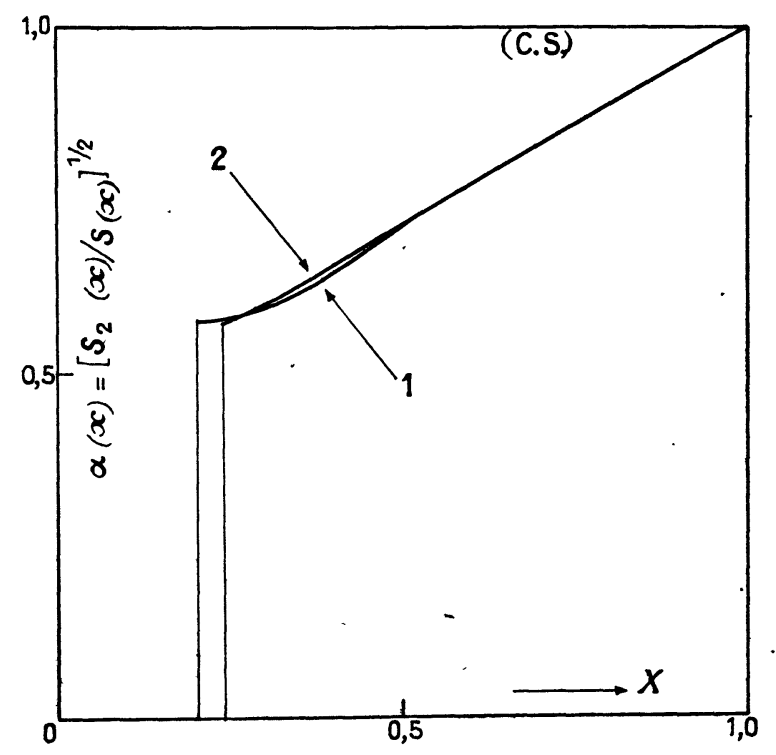

FIG. 4.

On a porté sur la figure 4, la quantité $\left[S_{2}(x) / S(x)\right]^{1 / 2}$ qui donne une information sur la largeur de la bande continue. Cette quantité est finie pour $x=x_{0}$ : dès qu'il apparaît des amas infinis, ils engendrent une bande de largeur finie. Les moments d'ordre supérieur se calculeraient de la même façon. 
Nous passons maintenant à l'étude de la conductivité de notre bande d'impuretés : les amplitudes $a_{i}$ décriventici des porteurs de courant qui ne peuvent se déplacer qu'en sautant d'atome $\mathbf{A}$ en atome A $(2 N \times f$ électron occupant $2 N x$ niveaux, le facteur 2 provenant du spin). Soit $\sigma(\omega)$ la conductivité (scalaire pour un cristal cubique) à la fréquence angulaire. Nous savons déjà que pour $x<x_{\mathrm{c}}$ la conductivité statique $\sigma(0)$ est nulle, et qu'elle est non nulle pour $x>x_{\mathbf{c}}$ (si l'on imagine une tranche de cristal placée entre deux électrodes planes finies, un amas infini a une probabilité 1 de rencontrer les deux électrodes). Nous allons préciser ces notions par un calcul de moments.

Nous supposons toujours nos porteurs indépendants. En outre, le problème étant plus difficile que celui de la densité de niveaux, nous le simplifions encore en nous plaçant dans la limite des hautes températures $\left(k_{\mathbf{B}} T \gg 1\right.$ dans nos unités). On peut alors en appliquant la méthode de Kubo [5], exprimer la conductivité en fonction des fluctuations de courant par une formule très compacte :

$$
\sigma(\omega)=\frac{f(1-f)}{3 k_{\mathbf{B}} T} \int_{-\infty}^{\infty} \mathrm{d} t \mathrm{e}^{-i \omega t} \operatorname{Tr}\{\boldsymbol{I}(0) . \boldsymbol{I}(t)\}
$$

I est l'opérateur courant à un électron

$$
\begin{aligned}
& \boldsymbol{I}=\frac{i e}{\hbar}[\varkappa, \boldsymbol{R}] \\
& \langle i|\boldsymbol{I}| j\rangle=\frac{i e}{h}\left(\boldsymbol{R}_{i}-\boldsymbol{R}_{i}\right) \quad \begin{array}{l}
\text { pour }(i) \text { et }(j) \text { voisins et } \\
\text { occupés par des atomes A. }
\end{array} \\
& =0 \\
& \text { (dans tous les autres cas) } \\
& \boldsymbol{I}(t)=\mathrm{e}^{i x t} \boldsymbol{I} \mathrm{e}^{-i x t} .
\end{aligned}
$$

Les moments d'ordre pair de $\sigma(\omega)$ (seuls sont nuls) sont donnés par

$$
\int \mathrm{d} \omega \omega^{2 n} \sigma(\omega)=\frac{2 n}{3} \frac{f(1-f)}{k_{\mathrm{B}} T} \operatorname{Tr}\left\{\boldsymbol{I} \cdot \frac{\mathrm{d}^{2 n}}{\mathrm{~d} t^{2 n}} \boldsymbol{I}\right\}_{t=0} .
$$

Les traces se calculent dans la représentation $|i\rangle$ en utilisant de façon répétée l'équation du mouvement déduite de (44)

$$
\frac{\mathrm{d}}{\mathrm{d} t} \boldsymbol{I}=\frac{i}{h}[\varkappa, \boldsymbol{I}] .
$$

Au voisinage de $x=1$ la contribution des amas finis étant négligeable, on peut postuler avec sécurité que $\sigma(\omega)$ est uen courbe continue. Si plus spécifiquement on prend une forme de Lorentz tronquée $\left(\sigma \cong \tau\left(1+\omega^{2} \tau^{2}\right)^{-1}\right.$ pour $|\omega|<\omega_{\mathrm{c}}$ et nulle au delà), on trouve par l'étude des moments d'ordre 2 et 4 , pour un cubique simple

$$
\begin{aligned}
& k \omega_{\mathbf{c}}=6,48 \\
& \tau \quad=0,34 \pi \frac{1}{y} .
\end{aligned}
$$

(Rappelons encore que notre unité d'énergie est l'élément de matrice de $x$ entre deux sites voisins.) L'intérêt de $\tau$ vient de ce qu'il représente une

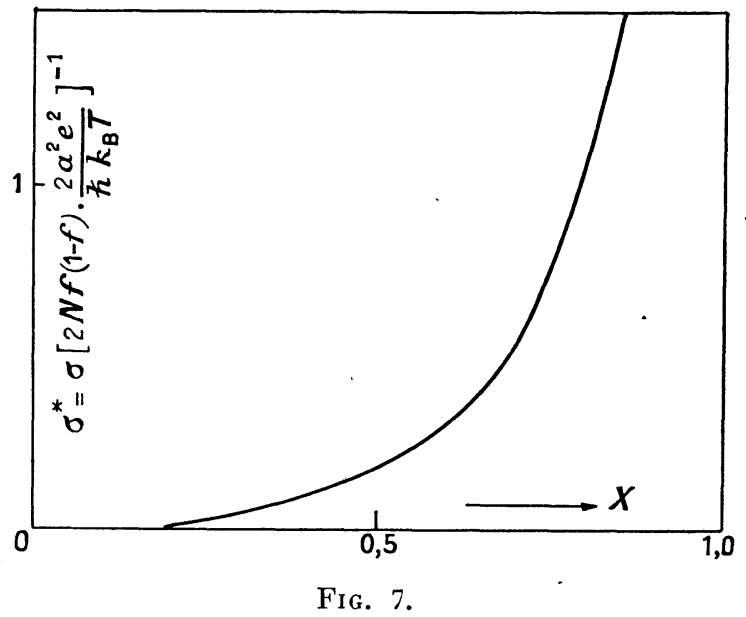

moyenne des temps de relaxation sur l'ensemble des niveaux. Aux concentrations intermédiaires, on doit, ici encore, éliminer le spectre discret par les mêmes méthodes. Le résultat d'un tel calcul (fait à la première approximation) est représenté sur la figure 5. Les valeurs numériques sont imprécises, mais l'aspect général doit être retenu, et en particulier l'annulation pour $x=x_{\mathbf{c}}$.

VI. Conclusions. - La propriété la plus remarquable exhibée par notre modèle est l'existence d'une concentration critique $x_{\mathbf{c}}$ qui résulte d'un effet purement géométrique, et qui sépare un domaine " macromoléculaire ". On retrouverait le même phénomène dans tous les cas tridimensionnels pourvu que la portée des couplages entre atomes actifs soit finie ( $x_{\mathbf{c}}$ étant d'autant plus faible que cette portée est grande).

Ces résultats sont à rapprocher de ceux d'Anderson [6] relatifs à une situation beaucoup plus générale. Le modèle d'Anderson diffère du nôtre en particulier par le fait que les niveaux des atomes A isolés sont supposés eux-mêmes aléatoires. L'exemple (schématisé à l'extrême) que nous avons choisi, permet de se faire une image correcte de l'existence d'une concentration critique et du comportement en deçà et au delà de cette concentration. L'existence d'un seuil critique pour les amas infinis a aussi été envisagée par Broadbent et Hammersley [7], dans une situation voisine où ce sont les couplages entre atomes qui sont aléatoires et non leur répartition: 
Transposés à notre situation, leurs théorèmes permettent d'écrire

$$
\begin{gathered}
\lim _{n \rightarrow \infty} \sum_{L} N_{n n}\left(R_{i}\right)=\mathrm{e}^{K n+0(n)} \\
x_{\mathbf{c}} \geqslant \mathrm{e}^{-K} .
\end{gathered}
$$

Du point de vue de l'analyse combinatoire, la confrontation de la méthode "optique " de la section II avec la méthode de trace de la section IV, conduit à des résultats remarquables, que nous résumons dans l'appendice.

Enfin, poúr en revènir aux problèmes physiques qui nous ont servi de point de départ, nous pouvons formuler les remarques suivantes :

1) Lorsque les atomes A sont des porteurs de moments magnétiques couplés ferromagnétiquement, le spectre des ondes de spin se déforme au fur et à mesure que l'on ajoute des atomes B, puis disparait pour $x=x_{c}$. Pour $x<x_{c}$, le système se comporte comme une collection d'amas finis d'atomes A et sa fonction de partition est donc une fonction régulière de la température : il n'y a pas de point de Curie bien défini dans ce domaine.

2) Lorsque les atomes A forment une bande d'impuretés dans un semi-conducteur comme $G e$ ou $S i$, il est tentant de relier $x_{c}$ à la concentration critique qui apparaît aux basses températures pour leur conductivité et pour le temps de relaxation spin réseau [8], [9]. (L'interprétation de cette dernière propriété repose sur le fait que des électrons itinérants subissent plus fortement l'action perturbatrice du réseau que des électrons liés à des atomes $\mathrm{A}$ isolés [10].)

Toutefois, nous ne pensons pas que ce problème puisse être résolu sans tenir compte des corrélations de Coulomb entre porteurs, qui ont été négligées ici sans justification, et qui pourraient bloquer la conductivité statique dans un domaine où celle-ci serait "géométriquement permise » [11].

Nous voudrions enfin remercier les Prs J. Friedel et A. Herpin pour plusieurs discussions sur ces questions, et le $\mathrm{D}^{\mathrm{r}} \mathrm{P}$. W. Anderson qui a eu l'amabilité de nous transmettre son manuscrit avant publication. Nous remercions d'autre part, $\mathrm{M}^{\mathrm{me}}$ Pillard et M. Grandidier qui ont effectué les calculs numériques.

Appendice 1. - Nous montrons ici comment l'approximation "optique " de la section II détermine certaines propriétés purement combinatoires des chemins tracés sur le réseau. Dans le domaine de validité de cette approximation, et pour les ordres inférieurs à $Y^{z}$, on peut associer à chaque vecteur d'onde $\boldsymbol{k}$ (d'énergie $\boldsymbol{E}(\boldsymbol{k})$ dans le cristal A pur) une énergie perturbée $E_{1}(\boldsymbol{k})$, et l'on a d'après (36)

$$
\overline{\bar{x}}_{1}^{n}=\sum_{p} N_{n p}(0) x^{p}=V_{0}(2 \pi)^{3} \int_{\text {zone }} d_{3} m E_{1}^{n}(\boldsymbol{m}) .
$$

Pour $x=1$, (48) restitue une formule classique de marche aléatoire. Si l'on prend la dérivée logarithmique de (48) on trouve :

$$
\begin{aligned}
& \bar{p}_{n} \equiv \frac{\sum_{p} N_{n p}(0) p}{\sum_{p} N_{n p}(0)} \\
& =n \frac{\int_{\text {zone }} \dot{d}_{3} m E^{n}(\boldsymbol{m})\left[\frac{\partial}{\partial x} \alpha n E_{1}(\boldsymbol{m})\right]_{x=1}}{\int_{\text {zone }} \mathrm{d}_{3} m E^{n}(\boldsymbol{m})}
\end{aligned}
$$

Pour $n \rightarrow \infty$ seules interviennent dans (49) les valeurs extrêmales de $|E(\boldsymbol{m})|$ qui sont

$$
|E(\boldsymbol{m})|=E(0)=Z \text {. }
$$

Nous nous limitons aux réseaux où ces valeurs ne sont obtenues qu'en un point de la zone (ex. : cfc) ou en des points de propriétés équivalentes à un changement de signe pour. $E$ (ex. : c. s.). On a alors

$$
\begin{aligned}
\lim _{n \rightarrow \infty} \frac{1}{n} \bar{p}_{n} & =\frac{1}{E(0)}\left[\frac{\partial}{\partial x} E_{1}(0)\right]_{x=1} \\
& =-\frac{K(Z)}{Z}
\end{aligned}
$$

où nous avons fait usage de (20). On peut calculer numériquement l'intégrale (21) qui conduit à $K(E)$. On trouve ainsi que (50) prend la valeur 0.702 pour un cubique simple.

Appendice 2. - On peut se demander si $x_{\mathrm{c}}$ n'est pas toujours égål à 1, c'est-à-dire si, dès qu'il existe un faible pourcentage d'atome $B$, les atomes A ne forment plus que des amas finis (quoique très grands).

En fait ceci n'a lieu que pour la chaîne linéaire. Soient en effet deux atomes A situés aux nœuds $(i)$ et $(j)$ d'un réseau à 2 dimensions, ou plus. Dans un domaine fini dè concentrations $x<1$, la probabilité $w$ pour que ces deux atomes appartiennent à un même amas n'est pas nulle, même lorsque $(i)$ et $(j)$ sont arbitrairement éloignés. Nous résumerons ici la démonstration de cette propriété sur le réseau carré plan. Soit $\left(i_{1}\right)\left(i_{2}\right) \ldots l\left(i_{N}\right)$ le (ou l'un des) plus court chemin reliant $(i)$ et $(j)$. Pour que $(i)$ et $(j)$ soient isolés, il faut que $(i)$ soit entouré d'une "enveloppe " d'atomes B n'enfermant pas $(j)$ ou réciproquement, ou qu'ils soient séparés par une même "enveloppe " d'atomes B de rang infini. De telles enveloppes forment elle-mêmes des amas d'atomes $\mathrm{B}$, au sens généralisé (couplages entre premiers ou seconds voisins dans notre cas). Chacune d'elles coupe le chemin $\left(i_{1}\right) \ldots\left(i_{\mathrm{N}}\right)$ en au moins un point $\left(i_{p}\right)$. Soit $\theta_{p}^{\prime}$ la probabilité pour 
que $\left(i_{p}\right)$ fasse partie d'une telle enveloppe. On a

$$
1-w<\sum_{1}^{N} \theta_{p}
$$

Toutes les enveloppes passant pas $\left(i_{p}\right)$ ont un rang supérieur ̀̀ $r(p)$, où $r$ est le plus petit des nombres $p$ et $N-p$. Donc $\theta_{p}$ est inférieur à la probabilité pour que $\left(i_{p}\right)$ appartienne à un amas généralisé quelconque d'atomes $\mathrm{B}$, de rang supérieur ou égal à $r(p)$. Par extension des raisonnements de la section III, on montre que

$$
\theta_{p}<\frac{(\alpha y)^{r}}{1-\alpha y}
$$

où $\alpha$ est un nombre caractéristique du réseau. Par conséquent

$$
\begin{gathered}
1-W<\frac{2}{(1-\alpha y)^{2}}\left[\alpha y-(\alpha y)^{N / 2}\right] \\
W>1-\frac{2 \alpha y}{(1-\alpha y)^{2}}
\end{gathered}
$$

quel que soit $N$. Par exemple pour $y<\frac{1}{4 \alpha}$, w est supérieur à $1 / q$. On pourrait évidemment, tout au long du calcul, chercher des majorantes plus serrées.

Manuscrit reçu le 7 février 1959.

\section{BIBLIOGRAPHIE}

[1] Watson (K. M.), Phys. Rev., 1953, 89, 575.

[2] Lippan (B.) et Senwinger (J.), Phys. Rev., 1950 , $79,669$.

[3] FRIEDEL (J.), Phil. Mag., 1952, 43, 153.

[4] De WItT (B. S.), Phys. Rev., 1956, 103, 1565.

[5] Kuвo (R.), Canad. J. Phys., 1956, 34, 1274.

[6] Anderson (P. W.), Phys. Reo., 1958, 109, 1492.
[7] Broadbent (S. R.) et Hammersley (J. M.), Proc. Camb. Phil. Soc., 1957, 53, 629.

[8] Conwell (E. M.), Phys. Rev., 1956, 103, 51.

[9] Feher (G.), Flecher (R. G.) et Gore (E. A.), Phys. Rev., 1955, 100, 784 .

[10] Bapdeen (J.), Pines (D.) et Slichter (C.), Phys. Rev., 1957, 106, 489.

[11] Mott (N. F.), Canad. J. Phys., 1956, 34, 1356. 